\title{
Surface melting of methane and methane film on magnesium oxide
}

\author{
N. Klipa \\ Department of Physics, University of Zagreb, Bijenička 32, 10000 Zagreb, Croatia \\ G. Bilalbegović \\ Department of Physics, University of Rijeka, Omladinska 14, 51000 Rijeka, Croatia \\ (to appear in Surface Science)
}

\begin{abstract}
Experiments on surface melting of several organic materials have shown contradictory results. We study the Van der Waals interactions between interfaces in surface melting of the bulk $\mathrm{CH}_{4}$ and interfacial melting of the $\mathrm{CH}_{4}$ film on the $\mathrm{MgO}$ substrate. This analysis is based on the theory of Dzyaloshinskii, Lifshitz, and Pitaevskii for dispersion forces in materials characterized by the frequency dependent dielectric functions. These functions for magnesium oxide and methane are obtained from optical data using an oscillator model of the dielectric response. The results show that a repulsive interaction between the solid-liquid and liquid-vapor interfaces exists for the bulk methane. We also found that the van der Waals forces between two solid-liquid interfaces are attractive for the $\mathrm{CH}_{4}$ film on the $\mathrm{MgO}$ substrate. This implies that the van der Waals forces induce the presence of complete surface melting for the bulk methane and the absence of interfacial melting for $\mathrm{CH}_{4}$ on the $\mathrm{MgO}$ substrate.

Keywords: Construction and use of effective interatomic interactions; Equilibrium thermodynamics and statistical mechanics; Dielectric phenomena; Surface melting; Wetting; Magnesium oxides; Alkanes; Solid-liquid interfaces;
\end{abstract}

\section{INTRODUCTION}

As the temperature is increased from below the bulk triple point, some solids begin to melt from their surfaces inward. If the thickness of the melted layer increases without limit as the temperature approaches the bulk triple point, then the solid is said to undergo surface melting [1:2]. The melting is incomplete if the thickness of the melted film remains finite as the temperature increases at all temperatures including the triple point. A special case of incomplete surface melting where the liquid film thickness is zero is sometimes referred to as surface nonmelting. For example, it was found that the $P b(110)$ surface melts. The $P b(111)$ 
surface does not melt, whereas $\mathrm{Pb}(100)$ exhibits incomplete melting. Therefore, incomplete or blocked melting appears on (100) fcc metal surfaces with an intermediate packing density between (110) and (111) [3,4]. It is characterized by a quasi-liquid film of the finite and small thickness. Surface melting was studied for several metals and other materials, such as ice, germanium, and multilayers of gases adsorbed on graphite [1,2]. High-temperature disordering for surfaces of several organic materials was also studied.

X-ray reflectivity measurements were used for high-temperature studies of the (100) face of caprolactam $\left(\mathrm{C}_{6} \mathrm{H}_{11} \mathrm{ON}\right)$ [5.6]. Initially a series of layering transition followed by prewetting was suggested, but later a surface hydration mechanism was proposed for temperature disordering of this surface. Chernov and Yakovlev studied surface melting for the (001) and (010) faces of biphenyl $\left(C_{12} H_{10}\right)$ using the ellipsometry technique [7]. They found that the thickness of a quasi-liquid layer at $0.5 \mathrm{~K}$ below the bulk melting point reaches $100 \AA$ for the (001) face and $300 \AA$ for the $(010)$ face. In contrast, de Jeu and coworkers using the synchrotron X-ray reflectivity measurements have shown the absence of surface melting for the (001) face of the bulk biphenyl up to $2 \mathrm{~K}$ below the melting point [8]. It was proposed that surface melting observed by Chernov and Yakovlev is induced by the roughness of the glass used in their ellipsometry experiment [8]. Surface properties of biphenyl, caprolactam, and other organic materials and organic thin films at high temperatures deserve further experimental and theoretical studies. Investigations of organic films are also important for applications, such as tribology and lubrication, corrosion inhibition, modification of the substrate optical properties, molecular electronics, and various industrial chemical processes.

Surface melting for a $\sim 10$ layer thick film of $\mathrm{CH}_{4}$ adsorbed on $\mathrm{MgO}(100)$ was studied by neutron scattering [9, 10]. It was found that the thickness of the quasi-liquid phase changes from one layer at $72 \mathrm{~K}$ to $\sim 6$ layers at $90.3 \mathrm{~K}$. (At atmospheric pressure the bulk melting point of methane is $90.66 \mathrm{~K}$, whereas the liquid-vapour transition occurs at $111.56 \mathrm{~K}$.) The neutron-scattering measurements of the translational diffusion coefficient above $72 \mathrm{~K}$ have shown that this quantity is in the $10^{-5} \mathrm{~cm}^{2} \mathrm{~s}^{-1}$ range, i.e., it has a value typical for mobility in quasi-liquid films. It was found that for a methane thick film $(\sim 20$ layers $)$ at $T=94 \mathrm{~K}$ (i.e., above the bulk melting temperature) almost all layers are liquefied [10]. Recently the layering and melting properties of methane on $\mathrm{MgO}(100)$ were studied between $70 \mathrm{~K}$ and 96 $\mathrm{K}$ using high-resolution adsorption isotherms and for the film thickness of up to five layers. [11]. Melting transition was observed at $80 \mathrm{~K}$ for the monolayer, and at $85 \mathrm{~K}$ for layers 2 through 4.

Related to the experiments on surface melting of bulk caprolactam and biphenyl, it would be interesting to study surface melting of a bulk methane. These studies should determine whether surface melting of the $\mathrm{CH}_{4}$ film on $\mathrm{MgO}$ is inherent feature of this organic material, or it is induced by the substrate. Interfacial melting is an appearance of a liquid film at the interface between one solid material and the substrate of the other material as the bulk melting point of the first material is approached [12,13. Therefore, surface melting is a special case of interfacial melting where the substrate is the vapor phase of the first material. In this work long-range interactions important for surface melting of a bulk methane, as well as for interfacial melting of a methane film on the $\mathrm{MgO}$ substrate are analyzed using the theory of Dzyaloshinskii, Lifshitz, and Pitaevskii (DLP) [14]. Our findings revealed that complete surface melting exists for the bulk methane. Because of an attractive interaction between interfaces melting is absent at the boundary between the $\mathrm{CH}_{4}$ film and the $\mathrm{MgO}$ 
substrate.

In the following the DLP theory of the van der Waals interaction between planar interfaces and its consequences for surface melting are described in Sec. II. The calculation of frequency dependent dielectric functions of $\mathrm{CH}_{4}$ and $\mathrm{MgO}$ is discussed in Sec. III. Results and discussion for the surface and interfacial melting problems are presented in Sec. IV. A summary and conclusions are given in Sec. V.

\section{SURFACE MELTING AND VAN DER WAALS FORCES}

The surface free energy per unit area for a system with a quasi-liquid film of the thickness $l$, at the temperature $T$ is [1,2,15]

$$
\mathcal{F}(l, T)=\gamma_{s l}+\gamma_{l v}+L_{m}\left(1-T / T_{m}\right) l+F_{s r}+F_{l r}
$$

In this equation $\gamma_{s l}$ and $\gamma_{l v}$ are the free energies of the solid-liquid and liquid-vapor interfaces, respectively, $L_{m}$ is the latent heat of melting per unit volume, and $T_{m}$ is the bulk melting temperature. The fourth term $F_{s r}$ represents the gain in interfacial energy associated with the wetting of the solid surface by a quasi-liquid film. This term is the result of shortrange interactions. Their contribution is oscillatory and decreases exponentially to zero for large $l$. Short-range forces produce a logarithmic temperature dependence of the liquid film thickness as the bulk melting temperature is approached. Long range van der Waals interactions are given by the last term $F_{l r}$ in Eq. (1). The contribution of the van der Waals forces for non-retarded interactions (where $l$ is much smaller than the wavelength of light for a characteristic excitations) is given by $H^{-2}$. The parameter $H$ is the Hamaker constant and gives information about the magnitude of the van der Waals interactions in different media [16]. (Sometimes $H^{\prime}=12 \pi H$ or $H^{\prime \prime}=-12 \pi H$ are called the Hamaker constant.) Surface melting occurs when $H>0$, i.e., when two interfaces (solid-liquid and liquid-vapour) repel each other. Then $F_{l r}(l)$ is monotonously decreasing function, or its minimum is not deep enough to bind the interface. The van der Waals contribution produces a power-law temperature dependence of the liquid film thickness as $T \rightarrow T_{m}$

$$
l=\left[\frac{L_{m}\left(T_{m}-T\right)}{2 H T_{m}}\right]^{-1 / 3} .
$$

For $H<0$ two interfaces attract each other and the melting is at most incomplete. Then a deep minimum exists on the free energy curve $F_{l r}$, and the interface is trapped there.

Dzyaloshinskii, Lifshitz, and Pitaevskii developed the theory of long-range dispersion interaction between macroscopic bodies [14,16]. The van der Waals interaction between interfaces is a consequence of electromagnetic fluctuations in a polarizable medium and the discontinuity of the dielectric function across the boundaries. In the DLP theory the force between interfaces (such as those shown in Fig. 1) is a function of the frequency-dependent dielectric functions $\epsilon(\omega)$ of materials in contact. In the following all media are taken as isotropic and continuous. The free energy per unit area of two media (1 and 2) separated by a film of thickness $l$ (media 3, see Fig. 1) is 14, 17, 12 


$$
\begin{array}{r}
F_{l r}(l, T)=\frac{k T}{8 \pi l^{2}} \sum_{m=0}^{\infty}, \int_{r_{m}}^{\infty} d x x\left(\ln \left(1-\frac{\left(x-x_{1}\right)\left(x-x_{2}\right)}{\left(x+x_{1}\right)\left(x+x_{2}\right)} \exp (-x)\right)\right. \\
\left.+\ln \left(1-\frac{\left(\epsilon_{2} x-\epsilon_{3} x_{2}\right)\left(\epsilon_{1} x-\epsilon_{3} x_{1}\right)}{\left(\epsilon_{2} x+\epsilon_{3} x_{2}\right)\left(\epsilon_{1} x+\epsilon_{3} x_{1}\right)} \exp (-x)\right)\right),
\end{array}
$$

where

$$
r_{m}=2 l \xi_{m}\left(\epsilon_{3}\right)^{1 / 2} / c
$$

and

$$
x_{1,2}=\left(x^{2}-r_{m}^{2}\left(1-\frac{\epsilon_{1,2}}{\epsilon_{3}}\right)\right)^{1 / 2} .
$$

The dielectric functions $\epsilon_{1}(\omega), \epsilon_{2}(\omega)$, and $\epsilon_{3}(\omega)$ are evaluated at imaginary frequencies $i \xi_{m}=$ $i(2 \pi k T / \hbar) m$. The functions $\epsilon(i \xi)$ are positive, real and decrease monotonically from the static dielectric function $\epsilon_{0}$ for $\xi=0$, to 1 as $\xi \rightarrow \infty$. In the above expressions $k$, $\hbar$, and $c$ are the Boltzmann and Planck constants and velocity of light, respectively. The prime

on the sum in Eq. (3) means that the term $m=0$ has to be multiplied with $\frac{1}{2}$. In the derivation of Eq. (3) retarded interactions were assumed. If retardation is ignored, then $c \rightarrow \infty, r_{m} \rightarrow 0$, and Eq. (1) simplifies to

$$
F_{l r}^{n r}(l, T)=\frac{k T}{8 \pi l^{2}} \sum_{m=0}^{\infty} \int_{0}^{\infty} d x x \ln \left(1-\frac{\left(\epsilon_{2}\left(i \xi_{m}\right)-\epsilon_{3}\left(i \xi_{m}\right)\right)\left(\epsilon_{1}\left(i \xi_{m}\right)-\epsilon_{3}\left(i \xi_{m}\right)\right)}{\left(\epsilon_{2}\left(i \xi_{m}\right)+\epsilon_{3}\left(i \xi_{m}\right)\right)\left(\epsilon_{1}\left(i \xi_{m}\right)+\epsilon_{3}\left(i \xi_{m}\right)\right)} \exp (-x)\right) .
$$

A common approach is to obtain an approximate value of $\epsilon(\omega)$ from the optical measurements 12,16 21. An oscillator model of the dielectric response is then used to generate data, i.e., the dielectric function is represented by

$$
\epsilon(\omega)=1+\sum_{i} \frac{f_{i}}{e_{i}^{2}-i \hbar \omega g_{i}-(\hbar \omega)^{2}}
$$

where $f_{i}, e_{i}$, and $g_{i}$ are fitting parameters. The DLP theory was recently applied to surface melting of ice [17,12], germanium, and several metals [18, 19]. Unfortunately, optical and dielectric properties of many organic materials are less studied than, for example of metals, semiconductors, or $\mathrm{H}_{2} \mathrm{O}$. As a consequence, we were not able to find necessary optical data for biphenyl and caprolactam, two organic materials for which experiments on surface melting were performed. In contrast, we found data for optical properties of organic material $\mathrm{CH}_{4}$ and dielectric substrate $\mathrm{MgO}$ [22, 23].

\section{DIELECTRIC FUNCTIONS OF METHANE AND MGO}

The complex dielectric functions $\epsilon(i \xi)$ were constructed using experimental data for optical constants of $\mathrm{CH}_{4}$ and $\mathrm{MgO}$ [22,23]. As suggested in Ref. [24] we minimized the objective function

$$
E=\sum_{j=1}^{N}\left[\left|\frac{\epsilon_{r}\left(\omega_{j}\right)-\epsilon_{r}^{e x p}\left(\omega_{j}\right)}{\epsilon_{r}^{e x p}\left(\omega_{j}\right)}\right|+\left|\frac{\epsilon_{i}\left(\omega_{j}\right)-\epsilon_{i}^{e x p}\left(\omega_{j}\right)}{\epsilon_{i}^{\exp }\left(\omega_{j}\right)}\right|\right]^{2}
$$


where $\epsilon_{r}(\omega)$ and $\epsilon_{i}(\omega)$ are the real and imaginary parts of a dielectric function given by Eq. (7), and 'exp' denotes experimental values taken from Refs. [22,23]. Therefore, the calculation was performed by finding a minimum of an objective function based on a simultaneous fitting of both real and imaginary parts of the dielectric function. We found that, in comparison with some other functions, the functional form in Eq. (8) is suitable for minimization. In principle, the most important factor in the minimization process is the quality of experimental data, i.e., the number of points and their distribution. It is known that the main contribution belongs to data in the UV region of the spectra [20,21]. The UV part of the spectrum for $M g O$ is represented with many points [23]. In contrast, in the UV spectra for the solid and liquid methane few points exist [22]. A smaller number of points gives more freedom in the choice of the parameters which minimize Eq. (8). Therefore, more local minima exist and they produce similar values of the objective function. This is a limitation for the calculation of dielectric properties of methane. Fortunately previous calculations for other materials have shown that the complex dielectric functions $\epsilon(i \xi)$ which enter expressions in the DLP theory are not very sensitive to the fitting procedure and the detailed experimental information. As suggested in Ref. [20] and followed by others [17,12], we used the constraint $\epsilon_{r}=n_{v i s}^{2}$, where $n_{v i s}$ is the refractive index in the visible part of the spectrum. The value $n_{v i s}$ is known with a great accuracy and fitting with this constraint gives better results. Objective functions were minimized using the simplex method 25,26. The accuracy of calculation was checked by changing minimization routines and also by calculating dielectric functions for other materials where extensive data are available 12,16 21.

Martonchik and Orton, motivated by astrophysical applications, recently calculated the optical constants of liquid and solid methane using the spectra from the literature [22]. Liquid methane was studied at the boiling and melting temperatures, whereas the solid phase I of methane was studied at the melting point and at $T=30 \mathrm{~K}$. (The solid phase I is the face centered cubic phase of methane which at atmospheric pressure exists for $T>20$ K.) Therefore, data obtained by Martonchik and Orton for the solid and liquid methane at the melting point were used to model the solid and liquid phase. For the vapor phase we put $\epsilon=1$. The static dielectric function of the gas methane is $\epsilon=1.000944$ [27]. The fits for $\mathrm{CH}_{4}$ were performed to match dielectric functions at zero frequency. These static dielectric functions are $\epsilon_{0}=1.67$ for the liquid methane, and $\epsilon_{0}=1.74$ for the solid methane [22]. The calculated complex dielectric functions of the solid and liquid methane are shown in Fig. 2. Corresponding fitting parameters are given in Tables I and II. The curves $\epsilon(i \xi)$ for the solid and liquid methane are close to each other, but do not cross. In similar calculation for surface melting of ice it was found that the complex dielectric functions of water and ice cross at $10 \mathrm{eV}$ (i.e., for $m \sim 205)$ [17, 12].

The detailed review of optical properties of $M g O$ was given by Roessler and Huffman [23]. The values of optical constants were tabulated in the range $(0.002-586) \mathrm{eV}$. Because of a large set of data we use only selected points. From these data the function $\epsilon(i \xi)$ for $\mathrm{MgO}$ was constructed. The static dielectric function of $M g O$ is $\epsilon_{0}=9.8$ [23]. Here, as in Ref. [28] where the fitting based on a double band was performed, we did not match the static dielectric function. The value of static dielectric function of $M g O$ is much larger than the average value of $\epsilon_{r}$ in the UV part of the spectrum. Additional problem is the existence of a close sharp peak at $\hbar \omega=7.8 \mathrm{eV}$. Therefore, the inclusion of the static dielectric function 
into the fit for $\mathrm{MgO}$ prevents a good behavior of the whole fitted curve. Fitting parameters for $M g O$ are given in Table III. The complex dielectric functions $\epsilon(i \xi)$ of $M g O$ is shown in Fig. 2. Our four-band result is almost the same as a double band calculation from Ref. [28.

\section{SURFACE AND INTERFACIAL MELTING}

\section{A. Bulk methane}

In surface melting problem the frequency dependent dielectric functions of solid, liquid (melt), and gas methane are respectively: $\epsilon_{1}=\epsilon_{s}(\omega), \epsilon_{3}=\epsilon_{l}(\omega)$, and $\epsilon_{2}=1$ (see Fig. 1). The integral in Eq. (3) was calculated numerically using the Gaussian method. The accuracy of the integration technique was checked by changing numerical procedures and also by reproducing the results for ice [17]. The calculated free energy as a function of liquid film thickness is shown in Fig. 3. The minimum is reached for $l \rightarrow \infty$. This functional form of $F(l)$ is typical for complete surface melting. We found that the Hamaker constant is $H=5.26 \times 10^{-22} \mathrm{~J}$. Similar values were calculated for metals with melted surfaces, such as $\mathrm{Pb}, \mathrm{Al}$, and $\mathrm{Au}[18]$.

\section{B. Methane film on $\mathrm{MgO}$}

In interfacial melting problem, and for interfaces shown in Fig. 1: $\epsilon_{1}=\epsilon_{M g O}(\omega)$ is dielectric function for $M g O, \epsilon_{2}=\epsilon_{s}(\omega)$ is for a solid phase, and $\epsilon_{3}=\epsilon_{l}(\omega)$ is for a liquid phase of methane. Therefore, we consider the solid $\mathrm{CH}_{4}$ - liquid $\mathrm{CH}_{4}-\mathrm{MgO}$ substrate system. The same numerical procedure as for the surface melting problem was applied. The free energy is shown in Fig. 4. The Hamaker constant is $H=-6.03 \times 10^{-22} \mathrm{~J}$. Therefore, the van der Waals forces produce an attractive interaction between interfaces and prevent interfacial melting. The functional form of $F(l)$ shown in Fig. 4 was not found for interfacial melting of ice on various substrates [12], but two other forms of $F(l)$ were calculated there. They correspond to complete and incomplete interfacial melting of ice on the particular substrate.

\section{SUMMARY AND CONCLUSIONS}

The interaction of interfaces present in surface melting of the bulk methane and interfacial melting of the $\mathrm{CH}_{4}$ film on the $\mathrm{MgO}$ substrate is analyzed within the Dzyaloshinskii, Lifshitz, and Pitaevskii theory of the van der Waals interaction. Frequency dependent dielectric functions for these materials are calculated from optical data. It was found that complete surface melting exists for methane. Our study is the first investigation of surface melting properties of the bulk methane. We also found that the van der Waals interactions do not produce interfacial melting of the $\mathrm{CH}_{4}$ film on the $\mathrm{MgO}$ substrate. Therefore, premelting observed in the experimental investigations of the $\mathrm{CH}_{4}$ films on the $\mathrm{MgO}$ substrate [9 11] is inherent feature of the bulk methane. Methane is till now the only organic material for which clear complete surface melting was found. The results obtained here depend on the quality of the optical measurements used to model dielectric functions. Nevertheless, 
these results shed light on the interaction between interfaces in surface and interfacial melting involving methane. These results suggest experimental studies of surface melting of the bulk methane, for example using the synchrotron X-ray reflectivity. In addition, ab initio molecular dynamics study of the high-temperature properties of methane surfaces is feasible, similar as a recent simulation of a high-pressure behavior for bulk $\mathrm{CH}_{4}$ [29]. It is important to do all necessary optical measurements for biphenyl and caprolactam, and then the calculation of the van der Waals interactions for these materials. There is a possibility that surface melting observed for biphenyl on the glass surface [7] is interfacial melting induced by the dielectric properties of the substrate. Till now the roughness of the glass and impurity induced interfacial melting were proposed to explain the results of Chernov and Yakovlev. A possibility of interfacial melting induced by the van der Waals forces for biphenyl on the glass also explains the absence of surface melting for the bulk biphenyl found using the X-ray reflectivity [8]. Recent atomic force microscopy study of the (001) surfaces of $n-C_{23} H_{48}$ paraffin crystals [30] shows that this technique gives a good opportunity to investigate melt growth on a molecular scale for surfaces of methane and other organic materials.

\section{ACKNOWLEDGMENTS}

This work has been carried out under the HR-MZT project 119206 - "Dynamical Properties of Surfaces". We would like to thank I. Kupčić and E. Ljubović for their help. 


\section{REFERENCES}

[1] J. F. van der Veen, B. Pluis, and A. W. Denier van der Gon, in Chemistry and Physics of Solid Surfaces VII, edited by R. Vanselow and R. F. Hove (Springer, Berlin, 1988), p. 455 .

[2] J. G. Dash, Contemp. Phys. 30, 89 (1989).

[3] H. M. van Pinxteren and J. W. M. Frenken, Surf. Sci. 275, 383 (1992).

[4] G. Bilalbegović and E. Tosatti, Phys. Rev. B 48, 11240 (1993).

[5] S. Chandavarkar, R. M. Geertman, and W. H. de Jeu, Phys. Rev. Lett. 69, 2384 (1992).

[6] G. C. L. Wong, W. H. de Jeu, G. Bögels, E. P. G. van den Berg, and P. Bennema, Phys. Rev. B 55, 1388 (1997).

[7] A. A. Chernov and V. A. Yakovlev, Langmuir 3, 635 (1987); A. A. Chernov and V. A. Yakovlev, Pis'ma Zh. Eksp. Teor. Fiz. 45, 130 (1987) [JETP Lett. 45, 160 (1987)].

[8] W. H. de Jeu, J. D. Shindler, S. Chandavarkar, R. M. Gertman, and K. Liang, Surf. Sci. 342, 341 (1995).

[9] M. Bienfait, Europhys. Lett. 4, 79 (1987).

[10] M. Bienfait, J. M. Gay, and H. Blank, Surf. Sci. 204, 331 (1988).

[11] A. Freitag and J. Z. Larese, Phys. Rev. B 62, 8360 (2000).

[12] L. A. Wilen, J. S. Wettlaufer, M. Elbaum, and M. Schick, Phys. Rev. B 52, 12426 (1995).

[13] D. Beaglehole and P. Wilson, J. Phys. Chem. 98, 8096 (1994).

[14] I. E. Dzyaloshinskii, E. M. Lifshitz, and L. P. Pitaevskii, Adv. Phys. 10, 165 (1961); V. L. Ginzburg, Theoretical Physics and Astrophysics, (in russian) (Nauka, Moskow, 1981), p. 350; Y. S. Barash and V. L. Ginzburg, Usp. Fiz. Nauk 143, 345 (1984) [Sov. Phys. Usp. 27, 467 (1984)].

[15] B. Pluis, D. Frenkel, and J. F. van der Veen, Surf. Sci. 239, 282 (1990); D. E. Sullivan and M. M. Telo da Gama, in: Fluid Interfacial Phenomena, edited by C. A. Croxton (Wiley, New York, 1986).

[16] J. N. Israelachivili, Intermolecular and Surface Forces, (Academic Press, London, 1985).

[17] M. Elbaum and M. Schick, Phys. Rev. Lett. 66, 1713 (1991).

[18] X. J. Chen, A. C. Levi, and E. Tosatti, Surf. Sci. 251/252, 641 (1991); Nuovo Cimento D 13, 919 (1991).

[19] A. DalCorso and E. Tosatti, Phys. Rev. B 47, 9742 (1993).

[20] V. A. Parsegian and G. H. Weiss, J. Colloid Interface Sci. 81, 285 (1981).

[21] D. B. Hough and L. R. White, Adv. Colloid Interface Sci. 14, 3 (1980).

[22] J. V. Martonchik and G. S. Orton, Appl. Optics 33, 8306 (1994).

[23] D. M. Roessler and D. R. Huffman, in "Handbook of optical constants of solids II", ed. by E. D. Palik, p. 919 (Academic Press, Boston, 1991).

[24] A. B. Djurišić, A. D. Rakić, and J. M. Elazar, Phys. Rev. E 55, 4797 (1997).

[25] W. H. Press, S. A. Teukolsky, W. T. Vetterling, and B. P. Flannery, Numerical Recipes in Fortran and Numerical Recipes in C, (Cambridge University Press, Cambridge, 1992/93).

[26] F. H. Walters, L. R. Parker, S. L. Morgan, and S. H. Deming, Sequential Simplex Optimization, http://www.multisimplex.com/simplexbook/index.htm, (MultiSimplex AB, 1997-2000). 
[27] Handbook of Chemistry and Physics, editied by R. C. Weast and M. J. Astle, (CRC Press, Boca Raton, 1979).

[28] E. S. Sabisky and G. H. Anderson, Phys. Rev. A 7, 790 (1973).

[29] F. Ancilotto, G. L. Chiarotti, S. Scandolo, and E. Tosatti, Science 275, 1288 (1997).

[30] M. Plomp, P. J. C. M. van Hoof, and W. J. P. van Enckevort, Surf. Sci. 448, 231 (2000). 


\section{FIGURES}

FIG. 1. Schematic representation for configuration of interfaces between media characterized by the frequency dependent dielectric functions.

FIG. 2. Complex dielectric functions. Dashed line for $M g O$ is the fit from Ref. [28]. The index $m$ is defined in Eqs. (3) and (6) in the text, and $m=1000$ corresponds to $48.8 \mathrm{eV}$. The contribution of various $m$-dependent terms in Eqs. (3) and (6) is obvious.

FIG. 3. The free energy per unit area ( $a_{0}$ is the Bohr radius) as a function of the film thickness $l$

for surface melting of the bulk methane. The dashed line shows the free energy without retardation, whereas the full line represents the result with retardation.

FIG. 4. The free energy vs film thickness for interfacial melting of a methane film on $\mathrm{MgO}$. Details as in Fig. 3. 


\section{TABLES}

TABLE I. Parameters used for an oscillator model of dielectric response for the solid methane using data from Ref. [22].

\begin{tabular}{lll}
\hline \hline$e_{i}(\mathrm{eV})$ & $f_{i}\left(\mathrm{eV}^{2}\right)$ & $g_{i}(\mathrm{eV})$ \\
\hline 10.0 & 10.0 & 1.8 \\
12.7 & 44.0 & 3.6 \\
14.4 & 64.0 & 3.5 \\
22.3 & 10.0 & 2.4 \\
23.1 & 23.0 & 6.8 \\
\hline \hline
\end{tabular}

TABLE II. Parameters used for an oscillator model of dielectric response for the liquid methane using data from Ref. [22].

\begin{tabular}{lll}
\hline \hline$e_{i}(\mathrm{eV})$ & $f_{i}\left(\mathrm{eV}^{2}\right)$ & $g_{i}(\mathrm{eV})$ \\
\hline 10.0 & 8.0 & 1.5 \\
12.8 & 42.0 & 3.5 \\
14.8 & 62.0 & 4.0 \\
22.7 & 8.0 & 2.4 \\
23.8 & 21.0 & 6.5 \\
\hline \hline
\end{tabular}

TABLE III. Parameters used for an oscillator model of dielectric response for solid $\mathrm{MgO}$ using data from Ref. [23].

\begin{tabular}{lll}
\hline \hline$e_{i}(\mathrm{eV})$ & $f_{i}\left(\mathrm{eV}^{2}\right)$ & $g_{i}(\mathrm{eV})$ \\
\hline 7.6 & 3.0 & 0.1 \\
10.8 & 150.0 & 3.5 \\
13.2 & 40.0 & 1.0 \\
17.0 & 130.0 & 4.0 \\
\hline \hline
\end{tabular}




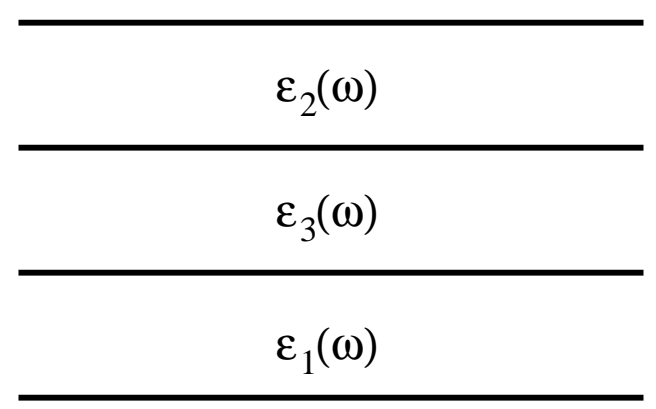

Fig.1 


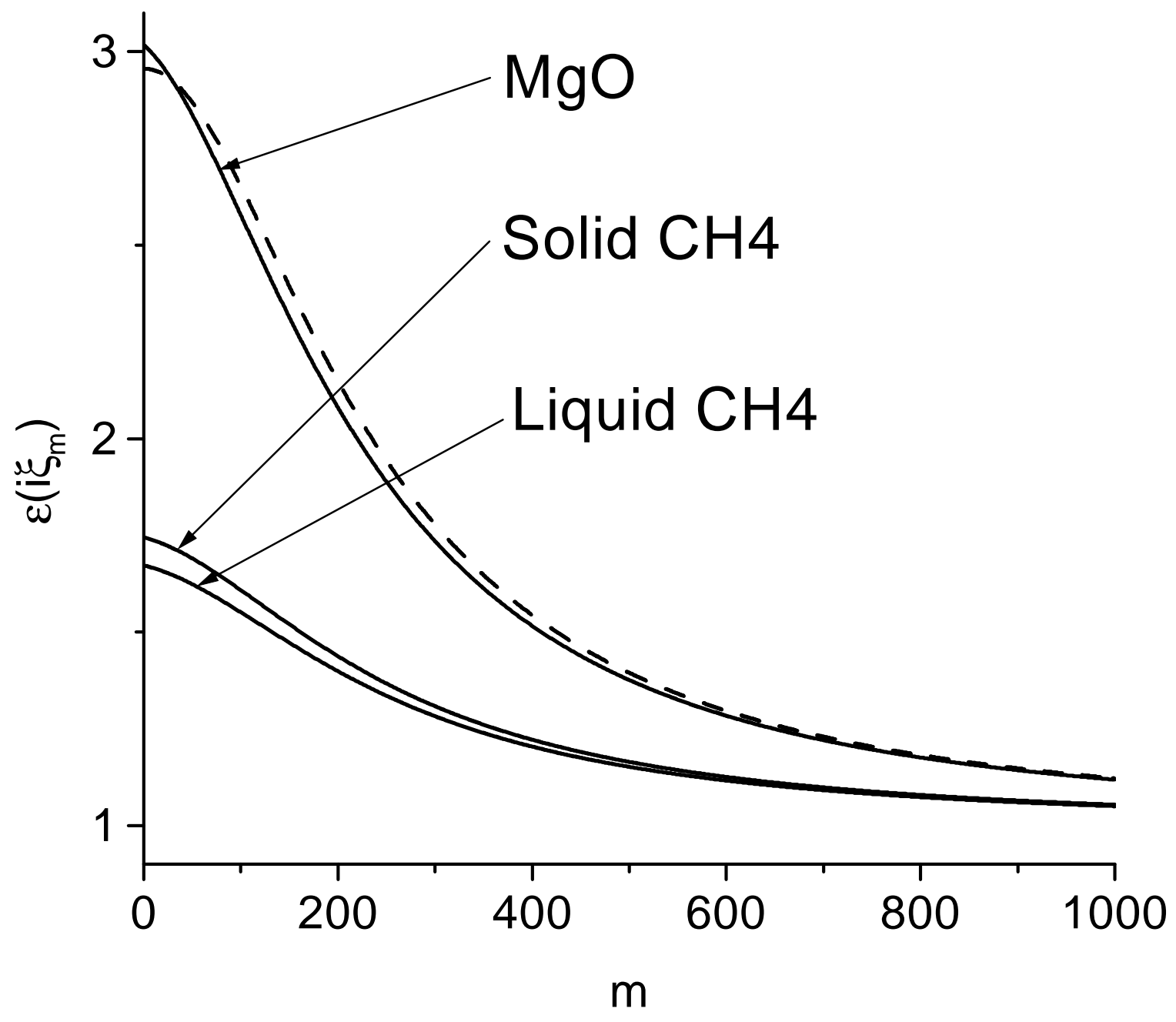

Fig.2 


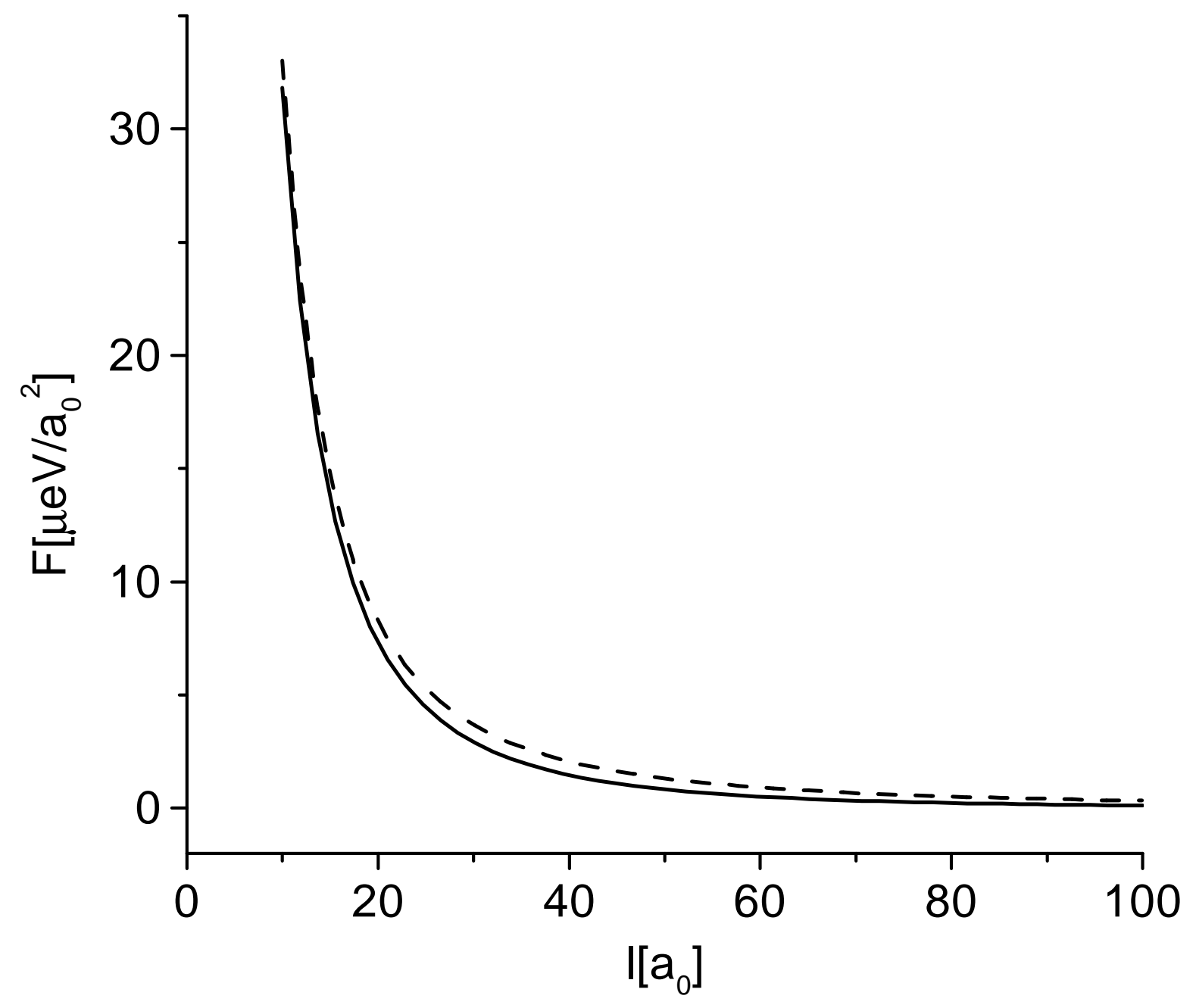

Fig.3 


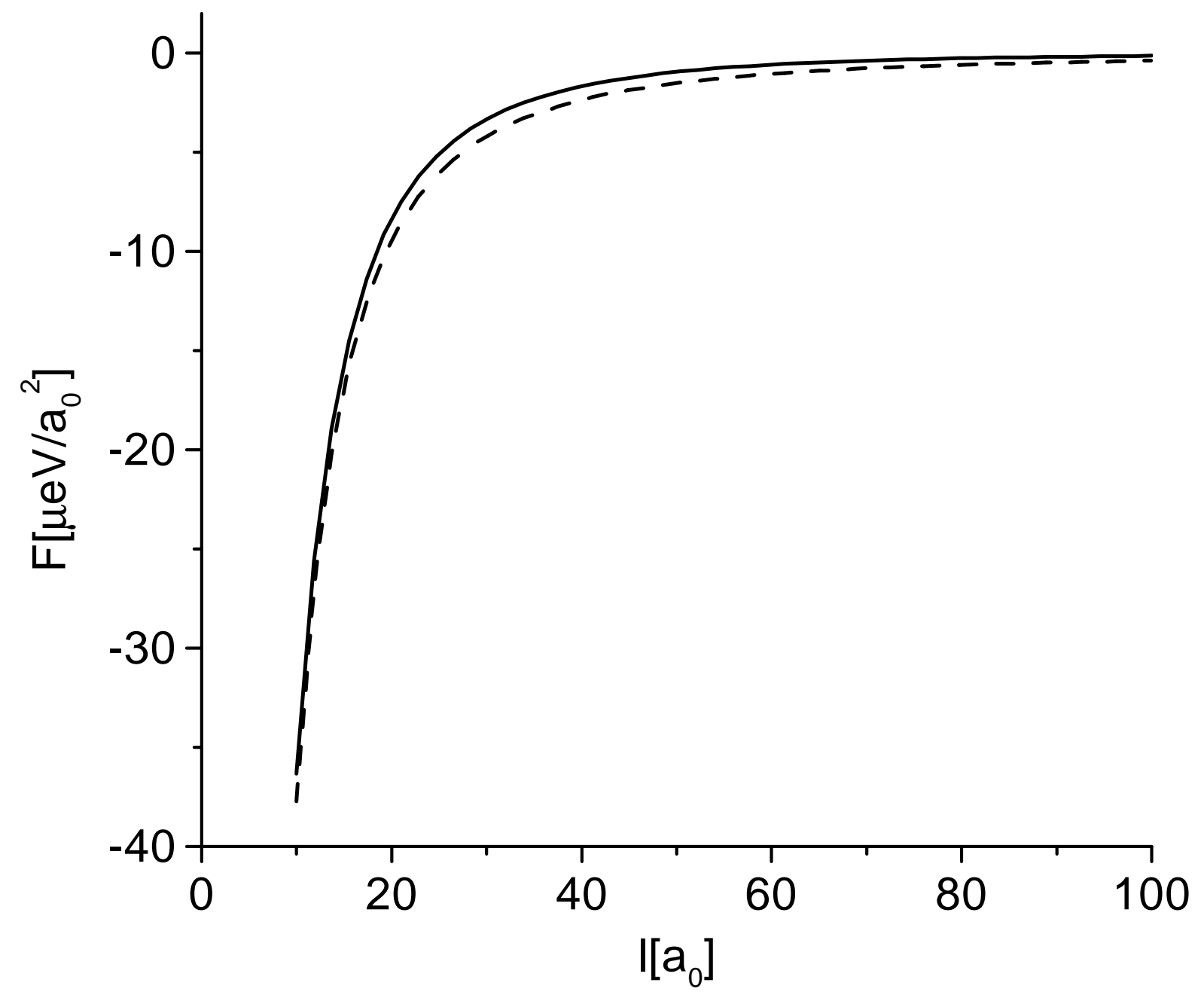

Fig.4 